\title{
Capacity Analysis of IRS-Based UAV Communications with Imperfect Phase Compensation
}

DOI:

10.1109/LWC.2021.3071059

\section{Document Version}

Accepted author manuscript

Link to publication record in Manchester Research Explorer

\section{Citation for published version (APA):}

Al-Jarrah, M., Alsusa, E., Al-Dweik, A., \& So, D. K. C. (2021). Capacity Analysis of IRS-Based UAV Communications with Imperfect Phase Compensation. IEEE Wireless Communications Letters.

https://doi.org/10.1109/LWC.2021.3071059

\section{Published in:}

IEEE Wireless Communications Letters

\section{Citing this paper}

Please note that where the full-text provided on Manchester Research Explorer is the Author Accepted Manuscript or Proof version this may differ from the final Published version. If citing, it is advised that you check and use the publisher's definitive version.

\section{General rights}

Copyright and moral rights for the publications made accessible in the Research Explorer are retained by the authors and/or other copyright owners and it is a condition of accessing publications that users recognise and abide by the legal requirements associated with these rights.

\section{Takedown policy}

If you believe that this document breaches copyright please refer to the University of Manchester's Takedown Procedures [http://man.ac.uk/04Y6Bo] or contact uml.scholarlycommunications@manchester.ac.uk providing relevant details, so we can investigate your claim.

\section{OPEN ACCESS}




\title{
Capacity Analysis of IRS-Based UAV Communications with Imperfect Phase Compensation
}

\author{
M. Al-Jarrah, Member, IEEE, E. Alsusa, Senior Member, IEEE, A. Al-Dweik, Senior Member IEEE, and Daniel \\ K. C. So, Senior Member, IEEE
}

\begin{abstract}
This paper presents the capacity analysis of unmanned aerial vehicles (UAVs) communications supported by flying intelligent reflecting surfaces (IRSs). In the considered system, some of the UAVs are equipped with an IRS panel that applies certain phase-shifts to the incident waves before being reflected to the receiving UAV. In contrast to existing work, this letter considers the effect of imperfect phase knowledge on the system capacity, where the phase error is modeled as a von Mises random variable with parameter $\kappa$. Analytical results, corroborated by Monte Carlo simulations, show that the achievable capacity is dependent on the phase error, however, the capacity loss becomes negligible at high signal-to-noise ratio (SNR) and when $\kappa \geq 6$.
\end{abstract}

Index Terms-Wireless backhauling, IRS, capacity, imperfect phase compensation, UAV, flying network.

\section{INTRODUCTION}

Because of their autonomy, flexibility, three dimensional mobility, and cost efficiency, unmanned aerial vehicles (UAVs) are receiving an increasing attention from industrial and academic researchers. UAVs have been considered for several applications such as surveillance, tracking, search and rescue missions, and remote sensing [1], [2]. They can also support existing cellular networks that experience temporary congestion or damage caused by environmental disasters such as earthquakes and storms. Recently, flying base-stations (BSs), or UAV based BS (UAV-BS), have been introduced to provide integrated access and backhaul (IAB) with wide coverage area, high capacity and ultimate connectivity. Moreover, the deployment of flying networks of various types of UAVs, including low altitude drones (LAD) and high altitude airships (HAA), is expected to provide reliable and high transmission rate communications as reported in [3]- $\mid 5]$. Some challenges for the deployment of LADs and HAAs are discussed in [3], where practical solutions are provided to ensure reliable connectivity.

Recently, intelligent reflecting surfaces (IRSs) have been proposed to control the wireless medium between transceivers. In IRS aided communications, a panel of programmable reflectors, which are able to apply phase shift to the incident waves and reflect them to the receiver, are used to enhance the signal quality

This work was supported by the European Union's Horizon 2020 Research and Innovation Programme through the Marie Sklodowska-Curie under Grant 812991. The work of A. Al-Dweik was supported by Khalifa University Competitive Internal Research Awards under Grant CIRA-2020-056.

M. A. Al-Jarrah, E. Alsusa and D. So are with the Department of Electrical and Electronic Engineering, University of Manchester, Manchester M13 9PL, U.K. (e-mail: \{mohammad.al-jarrah, e.alsusa, d.so\}@manchester.ac.uk).

A. Al-Dweik is with Center for Cyber Physical Systems (C2PS), Khalifa University, Abu Dhabi, P.O.Box 127788, UAE. (e-mail: arafat.dweik@ku.ac.ae).

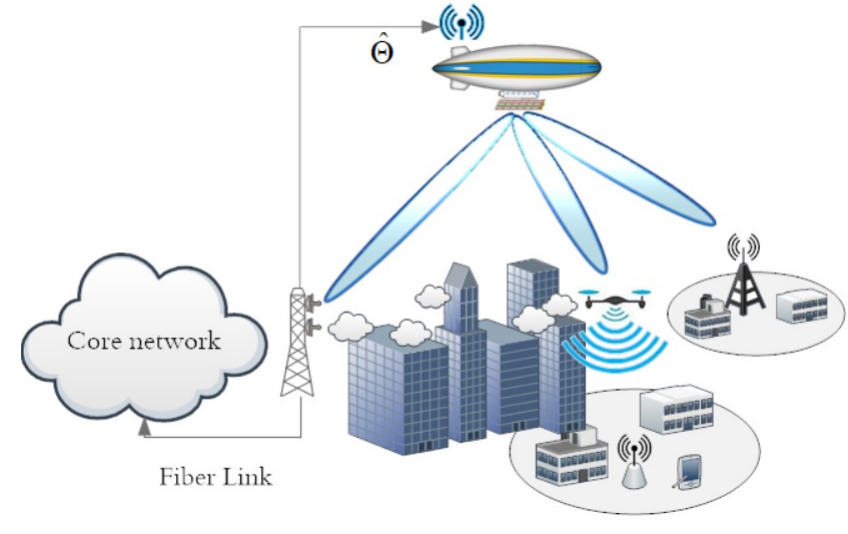

Fig. 1. IRS assisted flying networks.

at the receiver. The values of the phase-shifts for all reflectors are computed such that the reflected signals add coherently in the medium, and consequently the signal-to-noise ratio (SNR) can be significantly increased. The phase values are typically computed by the BS and sent to the programmable IRS through a control channel [6]-[8].

The achievable capacity and coverage probability with a limited number of reflectors are discussed in [9] under ideal and arbitrary phase compensation. The results in [9] showed that the capacity achieved with arbitrary phase shifts is very poor relative to the ideal case. However, ideal phase compensation is practically infeasible due to the phase estimation and quantization errors. Consequently, investigating the scenario of non-ideal phase compensation is necessary to explore the performance limits of IRS based communication systems [10]-[12]. In [10], the capacity limit of multiple-input multiple-output (MIMO) IRS systems is characterized by optimizing the reflection coefficients matrix of the IRS system aiming at maximizing the system capacity. In [11], the impact of a finite number of possible phase shifts on the achievable ergodic capacity (EC) is investigated, where the capacity bounds as a function of channel statistics are derived based on Jensen's inequality, and the phase quantization error is modeled as the derivative of the actual phase value. In [13], the bit error rate (BER) is derived where a statistical model is employed to characterize the phase compensation error caused by imperfect channel estimation and phase quantization. In addition to being considered separately, the synergy of IRSs and UAVs is considered as a promising solution for supporting reliable wireless communications in future generations such as the sixth generation (6G) and beyond [5], [14]. Fig. 1] depicts an example where IRS panels are attached to HAAs with hovering ability to support data exchange between small drones and a main BS. 
However, the wobbling of UAVs makes the assumption that the phase is perfectly compensated unrealistic. Consequently, evaluating the link capacity where IRS-UAV is incorporated with imperfect phase compensation is indispensable. Although similar system model is introduced in [5], the probability density function (PDF) of the received SNR, outage probability and BER have been analyzed, whereas the achievable EC has not been evaluated. Accordingly, the main contribution of this letter is the derivation of the achievable EC of flying IRS (FIRS) assisted UAV communications while considering the impact of imperfect phase compensation. Unlike [11], which considers the phase error as the derivative of the correct phase, we model the phase error using the von Mises PDF. The obtained results show that the phase compensation error has a significant impact on the achievable capacity at low values of $\kappa$ and SNR, however, the performance degradation becomes negligible at high values of SNR and $\kappa$.

The rest of the letter is organized as follows. Sec. II presents the system model of a flying network supported by an IRS. The derivation of the achievable capacity is parovided in Sec. III, while Sec. IV shows the numerical results. Conclusion and future work are provided in Section V.

\section{System MODEL}

As shown in Fig. 1, this work considers an IRS attached to HAA that is deployed to provide efficient wireless backhauling to terrestrial users. In addition, a flying BS represented as a drone is deployed to provide alternative line-of-sight ( $\mathrm{LoS})$ paths to users which may suffer from blockage. The IRS panel consists of $L$ elements each of which applies a phase shift $\hat{\theta}_{i}$, attenuates the signal by a coefficient $g_{i}$, and then reflects the signal to the destination drone. The phase compensation is assumed imperfect due to IRS phase noise and non-ideal phase estimation process, which becomes more challenging due to UAV wobbling. Given that the transmitted symbol is $s=|s| \mathrm{e}^{j \varphi}$, and the cascaded channel envelope is $\left|h_{i}\right|$ with total phase shift $\theta_{i}$, the received signal at the drone can be written as

$$
\tilde{r}(t)=|s| \sum_{i=1}^{L} A_{i} \cos \left(\omega_{\mathrm{c}} t+\varphi+\epsilon_{i}\right)+z(t)
$$

where $\omega_{\mathrm{c}}$ is the carrier angular frequency, $A_{i}=g_{i}\left|h_{i}\right|, \epsilon_{i}=$ $\hat{\theta}_{i}-\theta_{i}$ is the phase compensation error, and $z(t)$ is the additive white Gaussian noise (AWGN). Using the sinusoidal addition theorem (SAT) [15], $\tilde{r}(t)$ can be written as

$$
\tilde{r}(t)=|s| B_{L} \cos \left(\omega_{\mathrm{c}} t+\varphi+\zeta_{L}\right)+z(t), t \geq 0
$$

where and $\zeta_{L}$ are the equivalent channel envelope and phase, respectively,

$$
\begin{gathered}
B_{L}^{2}=\|\mathbf{A}\|^{2}+2 \sum_{L \geq j>k \geq 1} A_{j} A_{k} \cos \left(\epsilon_{j}-\epsilon_{k}\right), \\
\zeta_{L}=\tan ^{-1}\left(\sum_{i=1}^{L} A_{i} \sin \left(\epsilon_{i}\right) / \sum_{i=1}^{L} A_{i} \cos \left(\epsilon_{i}\right)\right)
\end{gathered}
$$

and $\|\cdot\|$ is the Euclidian norm. The elements of $\mathbf{A}=$ $\left[A_{1}, \ldots, A_{L}\right]$ depend on the channel model. For air-to-air chan- nels, the signal typically has a strong LoS component and a small number of weak scattered components; thus, the small scale fading of such channels follows the Rician distribution. However, according to experimental measurements, the Rician factor $K$ for ground-to-air and air-to-air channels is more than $15 \mathrm{~dB}$, and the received signal power may remain constant for long time periods [2], [4], [5], [16], [17]. Therefore, it can be assumed that the channel coefficients $A_{i}$ 's do not experience small scale fading, and that free space pathloss dominates the received signal power. Nevertheless, the obtained results in Sec. IV show that the constant fading coefficients model can be used to closely approximate the Rician fading channel with considerable values of $K$. It is worthy to note that the analysis provided here are valid for both BS-FIRS-UAV and BS-FIRSBS links with sufficiently high BSs, where these links are shown in Fig. 1 in elliptical shape. The von Mises, or circular normal, distribution is typically used to model the random phase error $\epsilon_{i}$ [13], where the PDF is given by

$$
f_{\epsilon_{i}}\left(\epsilon_{i}\right)=1 /\left(2 \pi I_{0}\left(\kappa_{i}\right)\right) \mathrm{e}^{\kappa_{i} \cos \left(\epsilon_{i}-\mu_{i}\right)}
$$

where $\mu_{i}$ and $\kappa_{i}$ are the mean and shape parameter of $\epsilon_{i}$. For unbiased estimators, the mean of $\epsilon_{i}$ is typically $\mu_{i}=0 \forall i$.

In slow fading channels, the accumulated phase offset $\zeta_{L}$ can be perfectly estimated by the receiving drone, and consequently the baseband representation of the received signal can be expressed as

$$
r=B_{L} s+z
$$

where $z \sim \mathcal{C N}\left(0, \sigma_{z}^{2}\right)$ is the AWGN.

\section{The AChievable CAPACITY}

The provided derivations consider a single user scenario. However, if the total number of reflectors is distributed among users with fixed assignment and orthogonal resource blocks are assigned to users [18], the analysis can be applied for multi-user system by adding the individual user's rates.

\section{A. Single Reflector $(L=1)$}

In this case, the received signal envelope is deterministic, and thus the instantaneous SNR is $\gamma_{1}=\frac{A_{1}^{2}}{\sigma_{z}^{2}}$. Consequently, the capacity normalized to the bandwidth $W$ is

$$
R_{1} \triangleq C_{1} / W=\log _{2}\left(1+\gamma_{1}\right)
$$

where $C_{1}$ indicates the capacity for the case $L=1$.

\section{B. Two Reflectors $(L=2)$}

Given the instantaneous normalized rate for the $L=2$ case $R\left(b_{2}\right)=\log _{2}\left(1+\frac{b_{2}^{2}}{\sigma_{z}^{2}}\right)$, where $b_{2}$ is the channel envelope when $L=2$, and the PDF of the signal envelope $f_{B_{2}}\left(b_{2}\right)$ in [5. eq. (22)], the average rate can be expressed as

$$
\begin{aligned}
\bar{R}_{2} & =\int_{0}^{\infty} R\left(b_{2}\right) f_{B_{2}}\left(b_{2}\right) d b_{2} \\
& =\frac{2}{\pi \tilde{\kappa}} \int_{\left|\mathcal{A}_{1}\right|}^{\mathcal{A}_{2}} \frac{b_{2} R\left(b_{2}\right) I_{0}\left(\sqrt{\left(\kappa_{1}-\kappa_{2}\right)^{2}+\frac{\kappa_{1} \kappa_{2}}{A_{1} A_{2}}\left(b_{2}^{2}-\mathcal{A}_{1}^{2}\right)}\right)}{\sqrt{-\left(b_{2}^{2}-\mathcal{A}_{2}^{2}\right)} \sqrt{\left(b_{2}^{2}-\mathcal{A}_{1}^{2}\right)}} d b_{2}
\end{aligned}
$$


where $\left|\mathcal{A}_{1}\right| \leq b_{2} \leq \mathcal{A}_{2}, \tilde{\kappa}=I_{0}\left(\kappa_{1}\right) I_{0}\left(\kappa_{2}\right), \mathcal{A}_{1}=A_{1}-A_{2}$ and $\mathcal{A}_{2}=A_{1}+A_{2}$. By using the infinite series definition for the modified Bessel function, $\bar{R}_{2}$ can be written as

$$
\begin{aligned}
& \bar{R}_{2}=\frac{2}{\pi \tilde{\kappa}} \int_{\left|\mathcal{A}_{1}\right|}^{\mathcal{A}_{2}} \frac{b_{2} R\left(b_{2}\right)}{\sqrt{-\left(b_{2}^{2}-\mathcal{A}_{2}^{2}\right)} \sqrt{\left(b_{2}^{2}-\mathcal{A}_{1}^{2}\right)}} \\
& \times \sum_{m=0}^{\infty} \frac{1}{2^{2 m}(m !)^{2}}\left(\left(\kappa_{1}-\kappa_{2}\right)^{2}+\frac{\kappa_{1} \kappa_{2}}{A_{1} A_{2}}\left(b_{2}^{2}-\mathcal{A}_{1}^{2}\right)\right)^{m} d b_{2} .
\end{aligned}
$$

To simplify the analysis, the binomial theorem is applied to find the algebraic expansion for the last term of the integrand in (9), and thus $\bar{R}_{2}$ can be expressed as

$$
\begin{aligned}
\bar{R}_{2}=\frac{2}{\pi \tilde{\kappa}} \sum_{m=0}^{\infty} \frac{1}{2^{2 m}(m !)^{2}} \sum_{n=0}^{m} \mathcal{T}_{m, n} \\
\times \int_{\left|\mathcal{A}_{1}\right|}^{\mathcal{A}_{2}} b_{2} R\left(b_{2}\right) \frac{\left(b_{2}^{2}-\mathcal{A}_{1}^{2}\right)^{n-0.5}}{\sqrt{-\left(b_{2}^{2}-\mathcal{A}_{2}^{2}\right)}} d b_{2}
\end{aligned}
$$

where $\mathcal{T}_{m, n}=\left(\begin{array}{c}m \\ n\end{array}\right)\left(\kappa_{1}-\kappa_{2}\right)^{2(m-n)}\left(\frac{\kappa_{1} \kappa_{2}}{A_{1} A_{2}}\right)^{n}$.

Using the integration by substitution rule with $y=b_{2}^{2}-\mathcal{A}_{1}^{2}$, and the logarithmic identity $\log _{k} x=\frac{\ln x}{\ln k}$ yields

$$
\begin{aligned}
\bar{R}_{2}= & \frac{1}{\pi \tilde{\kappa} \ln 2} \sum_{m=0}^{\infty} \frac{1}{2^{2 m}(m !)^{2}} \sum_{n=0}^{m} \mathcal{T}_{m, n} \\
& \times \int_{0}^{\mathcal{A}_{3}^{2}} \frac{y^{n-0.5}}{\sqrt{-y+\mathcal{A}_{3}^{2}}} \ln \left(\frac{y}{\sigma_{z}^{2}}+\frac{\sigma_{z}^{2}+\mathcal{A}_{1}^{2}}{\sigma_{z}^{2}}\right) d y
\end{aligned}
$$

where $\mathcal{A}_{3}=\sqrt{4 A_{1} A_{2}}$.

The integral in (11) can be solved using [19, 2.6.10.31, pp. 502], and consequently $\bar{R}_{2}$ can be found as

$$
\begin{gathered}
\bar{R}_{2}=\frac{1}{\pi \tilde{\kappa} \ln 2} \sum_{m=0}^{\infty} \frac{1}{2^{2 m}(m !)^{2}} \sum_{n=0}^{m} \mathcal{T}_{m, n} \\
\left\{\mathcal{A}_{3}^{2 n} \ln (\nu) \mathrm{B}(n+0.5,0.5)+\frac{\mathcal{A}_{3}^{2(n+1)}}{\sigma_{z}^{2} \nu} \mathrm{B}(n+1.5,0.5)\right. \\
\left.\quad \times{ }_{3} F_{2}\left([n+1.5,1,1],[2, n+2],-\frac{\mathcal{A}_{3}^{2}}{\nu \sigma_{z}^{2}}\right)\right\}
\end{gathered}
$$

where $\nu=\frac{\sigma_{z}^{2}+\mathcal{A}_{1}^{2}}{\sigma_{z}^{2}}$ and $\mathrm{B}(a, b) \triangleq \frac{\Gamma(a) \Gamma(b)}{\Gamma(a+b)}$ is the beta function. C. Three Reflectors $(L=3)$

As reported in [5], the PDF $f_{B_{3}}\left(b_{3}\right)$ does not have a closedform expression. Therefore, the capacity will be evaluated using the same approach of $L=2$, but the integrals will be solved numerically.

\section{Central Limit Theorem (CLT) for $L \geq 4$}

Since the derivation of closed-form expressions for the PDF when $L \geq 4$ is not feasible, the CLT is invoked. With the aid of SAT, $B_{L}^{2}$ in 3 can be rewritten as $B_{L}^{2}=B_{L, \mathcal{I}}^{2}+B_{L, \mathcal{Q}}^{2}$, where $B_{L, \mathcal{I}}=\sum_{i=1}^{L} A_{i} \cos \epsilon_{i}$ and $B_{L, \mathcal{Q}}=\sum_{i=1}^{L} A_{i} \sin \epsilon_{i}$. Since $A_{i}$ and $\epsilon_{i}$ are independent $\forall i$, CLT can be applied for large values of $L$ to evaluate the distributions of $B_{L, \mathcal{I}}$ and $B_{L, \mathcal{Q}}$. Accordingly, the distribution functions of $B_{L, \mathcal{I}}$ and $B_{L, \mathcal{Q}}$ can be found as $\mathcal{N}\left(\mu_{\mathcal{I}}, \sigma_{\mathcal{I}}^{2}\right)$ and $\mathcal{N}\left(\mu_{\mathcal{Q}}, \sigma_{\mathcal{Q}}^{2}\right)$, respectively. By noting that $\mathrm{E}\left[\cos \left(n \theta_{i}\right)\right]=\frac{I_{n}\left(\kappa_{i}\right)}{I_{0}\left(\kappa_{i}\right)}$ and $\mathrm{E}\left[\sin \left(n \theta_{i}\right)\right]=0$ [5. Appendix I], $\mu_{\mathcal{Q}} \triangleq \mathrm{E}\left[B_{\mathcal{Q}}\right]=0$ can be obtained whereas $\mu_{\mathcal{I}}$ can be found as $\mu_{\mathcal{I}}=\mathrm{E}\left[B_{\mathcal{I}}\right]=\sum_{i=1}^{L} A_{i} \frac{I_{1}\left(\kappa_{i}\right)}{I_{0}\left(\kappa_{i}\right)}$. The second moment for $B_{\mathcal{I}}$ can be evaluated as $E\left[B_{\mathcal{I}}^{2}\right]=\mathrm{E}\left[\sum_{i=1}^{L} A_{i} \cos \phi_{i}\right]^{2}$. Using the expansion for squared summation and, then evaluating the expected value yields

$$
\mathrm{E}\left[B_{\mathcal{I}}^{2}\right]=\sum_{i=1}^{L} \frac{A_{i}^{2}}{2}\left(1+\frac{I_{2}\left(\kappa_{i}\right)}{I_{0}\left(\kappa_{i}\right)}\right)+2 \sum_{i<j}^{L} A_{i} A_{j} \frac{I_{1}\left(\kappa_{i}\right) I_{1}\left(\kappa_{j}\right)}{I_{0}\left(\kappa_{i}\right) I_{0}\left(\kappa_{j}\right)} .
$$

Similarly, $\mathrm{E}\left[B_{\mathcal{Q}}^{2}\right]$ can be derived as

$$
\mathrm{E}\left[B_{\mathcal{Q}}^{2}\right] \triangleq \mathrm{E}\left[\sum_{i=1}^{L} A_{i} \sin \phi_{i}\right]^{2}=\sum_{i=1}^{L} \frac{A_{i}^{2}}{2}\left(1-\frac{I_{2}\left(\kappa_{i}\right)}{I_{0}\left(\kappa_{i}\right)}\right) .
$$

Consequently, $\sigma_{\mathcal{I}}^{2}$ and $\sigma_{\mathcal{Q}}^{2}$ can be found using the well known formula $\sigma_{\mathcal{I} \mid \mathcal{Q}}^{2} \triangleq \mathrm{E}\left[B_{\mathcal{I} \mid \mathcal{Q}}^{2}\right]-\mathrm{E}^{2}\left[B_{\mathcal{I} \mid \mathcal{Q}}\right]$.

Using the definition of the correlation coefficient, and then employing the product of two summations rule to find $\mathrm{E}\left[B_{L, \mathcal{I}} B_{L, \mathcal{Q}}\right]$, the correlation between $B_{L, \mathcal{I}}$ and $B_{L, \mathcal{Q}}$ is

$$
\rho_{\mathcal{I}, \mathcal{Q}}=\frac{1}{\sigma_{\mathcal{I}} \sigma_{\mathcal{Q}}} \sum_{i=1}^{L} \sum_{j=1}^{L} A_{i} A_{j} \mathrm{E}\left[\cos \epsilon_{i} \sin \epsilon_{j}\right] .
$$

For $i \neq j, \mathrm{E}\left[\cos \epsilon_{i} \sin \epsilon_{j}\right]=\mathrm{E}\left[\sin \epsilon_{j}\right] \mathrm{E}\left[\cos \epsilon_{i}\right]=0$. On the other hand, when $i=j$, the trigonometric identity $\cos \epsilon_{i} \sin \epsilon_{i}=0.5 \sin \left(2 \epsilon_{i}\right)$ can be applied, which implies that $\mathrm{E}\left[\cos \epsilon_{i} \sin \epsilon_{j}\right]=0$ as well . Since $B_{L, \mathcal{I}}$ and $B_{L, \mathcal{Q}}$ are Gaussian distributed according to CLT and they are uncorrelated, $B_{L, \mathcal{I}}$ and $B_{L, \mathcal{Q}}$ are independent random variables. For a special case when the phase error is uniformly distributed, $\kappa=0, \mu_{\mathcal{I}}=$ $\mu_{Q}=0$ and $\mathrm{E}\left[B_{\mathcal{I}}^{2}\right]=\mathrm{E}\left[B_{\mathcal{Q}}^{2}\right]=\sum_{i=1}^{L} A_{i}^{2} / 2$. Therefore, the propagation environment follows Rayleigh channel model when $\kappa=0$. For the general case when $\kappa>0, B_{L}$ is the envelope of a complex Gaussian with different values for the variance of the in-phase and quadrature components with one of the components has non-zero mean. The PDF of such random variable is given in [20, eq. (8)]. However, the form provided in [20] contains infinite sum of modified bessel functions product with different orders, which makes the distribution untraceable. An accurate approximation for the PDF of $\left|B_{L}\right|^{2}$ has been derived in [13], where the PDF is approximated as Gamma random variable with shape parameter $\alpha=\mu_{I}^{2} / 4 \sigma_{\mathcal{I}}^{2}$ and inverse scale factor $\beta=1 / 4 \sigma_{\mathcal{I}}^{2}$. By denoting $y=B_{L}^{2}$ and using the gamma distribution function, the achievable EC can be expressed as

$$
\begin{aligned}
\bar{R}_{\mathrm{CLT}} & =\int_{0}^{\infty} R\left(b_{L}\right) f_{B_{L}}\left(b_{L}\right) d b_{L} \\
& =\frac{\beta^{\alpha}}{\Gamma(\alpha) \ln 2} \int_{0}^{\infty} y^{\alpha-1} \mathrm{e}^{-\beta y} \ln \left(1+\frac{y}{\sigma_{z}^{2}}\right) d y .
\end{aligned}
$$

By using [19. eq. (2.6.23.4), pp. 530], $\bar{R}_{\mathrm{CLT}}$ can be found in 

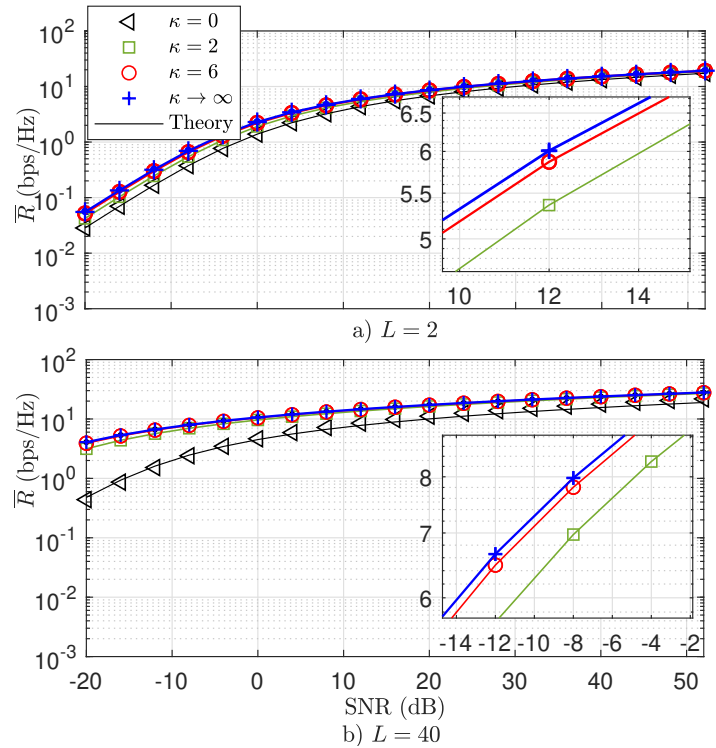

Fig. 2. The achievable $\bar{R}$ for $L=\{2,40\}$ for different values of $\kappa$, where $A_{i}=1 \forall i$.

closed-form as

$$
\begin{gathered}
\bar{R}_{\mathrm{CLT}}=\frac{\beta^{\alpha}}{\Gamma(\alpha) \ln 2}\left(\frac{\pi \sigma_{z}^{2 \alpha}{ }_{1} F_{1}\left(\alpha ; \alpha+1 ; \beta \sigma_{z}^{2}\right)}{\alpha \sin (\alpha \pi)}-\Gamma(\alpha) \beta^{-\alpha}\right. \\
\left.\times\left\{\ln \left(\beta \sigma_{z}^{2}\right)-\Psi(\alpha)+\frac{\beta \sigma_{z}^{2}}{1-\alpha}{ }_{2} F_{2}\left([1,1],[2,2-\alpha], \beta \sigma_{z}^{2}\right)\right\}\right)
\end{gathered}
$$

where $\Psi(\cdot)$ is the digamma function which is defined as $\Psi(\alpha)=\Gamma(\alpha) / \Gamma^{\prime}(\alpha)$.

For high SNR, $\frac{y}{\sigma_{z}^{2}} \gg 1$ and thus $\bar{R}_{\mathrm{CLT}}$ can be reduced to

$$
\bar{R}_{\mathrm{CLT}, \mathrm{H}}=\frac{\beta^{\alpha}}{\Gamma(\alpha) \ln 2} \int_{0}^{\infty} y^{\alpha-1} \mathrm{e}^{-\beta y} \ln \left(\frac{y}{\sigma_{z}^{2}}\right) d y .
$$

By using the logarithmic identity $\ln \frac{x}{y}=\ln x-\ln y$ and evaluating the resulting integral using [19, eq. (2.6.21.2), pp. 527] and [19, eq. (2.3.3.1), pp. 322], $\bar{R}_{\mathrm{CLT}}$ can be found as

$$
\bar{R}_{\mathrm{CLT}, \mathrm{H}}=\left(\Psi(\alpha)-\ln \beta-\ln \sigma_{z}^{2}\right) / \ln 2 .
$$

By comparing the derived $\bar{R}_{\mathrm{CLT}}$ in 19 with the formula in (17), it can be realized that [19] is more tractable and has less computational complexity.

\section{NuMERICAL RESULTS}

This section presents the achievable capacity for the considered system model. The analytical results obtained from the derived formulae are compared to Monte Carlo simulation results with $10^{7}$ realizations. To evaluate the impact of phase error compensation, the results are also compared to the cases of ideal and arbitrary phase compensation, which are respectively referred as $\kappa \rightarrow \infty$ and $\kappa=0$. The average transmission power is normalized to unity, and the SNR in $\mathrm{dB}$ is defined as $\mathrm{SNR}=$ $-10 \log _{10}\left(\sigma_{z}^{2}\right)$. Unbiased phase estimators with equal variance are considered, i.e., $\mu=0$ and $\kappa_{i}=\kappa \forall i$. For the infinite summation in (13), the first 30 terms have been considered.

Fig. 2 a shows the achievable normalized EC $\bar{R}$ in bps/Hz for
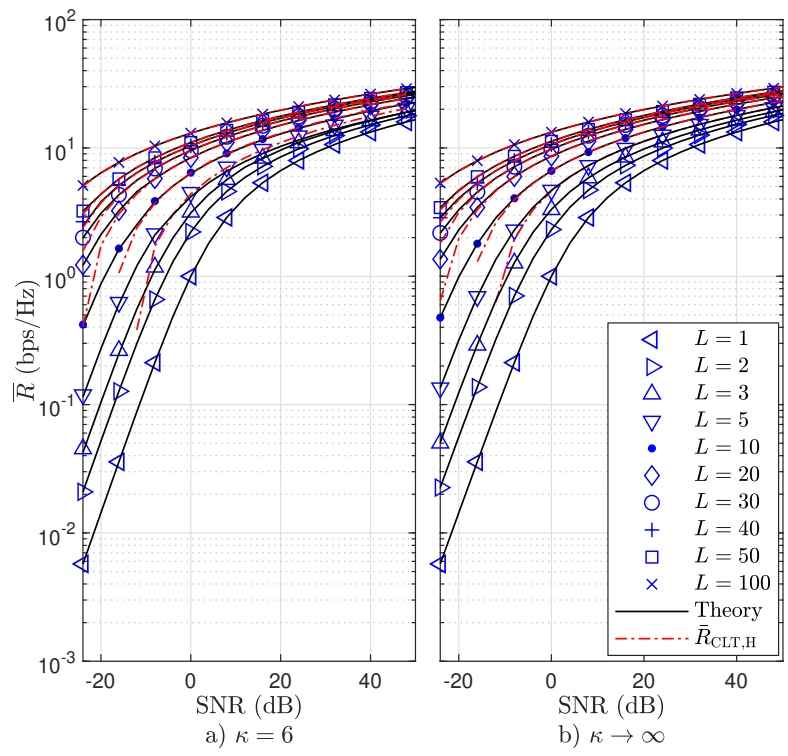

Fig. 3. The achievable $\bar{R}$ for different numbers of reflectors $L, A_{i}=1 \forall i$.

different values of $\kappa$ when the number of reflectors is $L=2$ and $A_{1}=A_{2}=1$. As can be observed from the figure, the derived equation for $\bar{R}_{2}$ matches the simulation results. As expected, the phase compensation errors negatively affect the achievable rate, and as $\kappa$ increases, i.e., channel estimation and compensation improves, the achievable rate improves. For example, when the SNR is $0 \mathrm{~dB}$, the capacity achieved with $\kappa \rightarrow \infty$, ideal phase shift, is about 1.7 times the capacity when $\kappa=0$, i.e., arbitrary phase compensation. However, the capacity loss decreases as $\kappa$ increases. For example, the capacity loss is less than $0.5 \mathrm{~dB}$ when $\kappa=6$ as compared to the ideal case. Moreover, the figure shows that the effect of $\kappa$ becomes negligible when $\kappa \geq 6$, which implies that near-ideal performance for $L=2$ can be achieved by designing a phase estimation and compensation processes satisfying the condition $\kappa \geq 6$.

Fig. $2 p$ shows the achievable $\bar{R}$ for different values of $\kappa$ when the number of reflectors $L=40$, where $A_{i}=1 \forall i$. The obtained results show a perfect match for the derived CLT based equation for a wide range of $\kappa$ values, except for the case of $\kappa=0$. Similar to Fig. 2al, the impact of $\kappa$ decreases as its value increases and becomes negligible when $\kappa \geq 6$. However, by comparing Fig. 2p with Fig. 2a, it can be realized that when $L=40$, the arbitrary phase compensation causes significant capacity loss as compared to the ideal case. For example the difference between the arbitrary and ideal scenarios in Fig. 2p is about $20 \mathrm{~dB}$ at $\bar{R}=10 \mathrm{bps} / \mathrm{Hz}$.

Figs. 3a and 3p show the impact of increasing the number of reflectors $L$ on the achievable rate $\bar{R}$ when the phase compensation error parameter $\kappa=6$ and $\kappa \rightarrow \infty$, respectively. As can noticed, a perfect match between simulation and theoretical results is obtained, and the asymptotic capacity derived in (19) for $L \geq 4$ converges to the exact formula in 17 when $\bar{R} \gtrsim 3$ bps/Hz. By comparing Fig. 33 with Fig. 3b, it can be observed that the effect of phase compensation errors is negligible when $\kappa=6$. It is worth noting that the case of $L=1$ is not affected by 


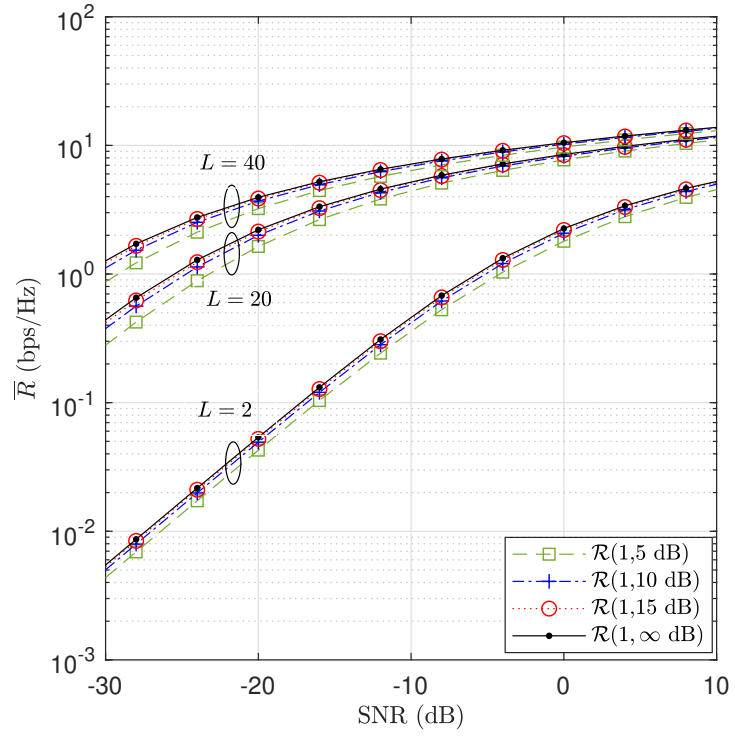

Fig. 4. The achievable $\bar{R}$ with Rician fading channel $\mathcal{R}(\Omega, K)$ for $\Omega=1$ and different values of $K$, where $\kappa=10$.

$\kappa$, as discussed in Sec. III. The figure shows that $\bar{R}$ significantly improves by increasing $L$. For example, at $\mathrm{SNR}=0 \mathrm{~dB}$, the value of $\bar{R}$ increases by 10 -fold when $L$ is increased from 1 to 50 . Moreover, by comparing $L=1$ with other cases, it is realized that deploying IRS with phase compensation error statistics of $\kappa=6$ can enhance the system capacity considerably.

Fig. 4 shows the achievable normalized rate $\bar{R}$ for double Rician, i.e., $\left\{\left|\hbar_{i}\right|,\left|h_{i}\right|\right\} \sim \mathcal{R}(\Omega, K)$ for $\Omega=1$ and different values of the Rician factor $K$. It is noteworthy that the case of $K \rightarrow \infty$ is equivalent to the deterministic channel scenario with $A_{i}=1 \forall i$. The figure shows that the impact of the Rician factor is negligible when $K \geq 15 \mathrm{~dB}$. For example, the achievable rate when $K=15 \mathrm{~dB}$ is almost the same as the deterministic channel scenario, $(K \rightarrow \infty)$. In addition, the impact of $K$ becomes less important for small values of $L$ and the rate is mainly determined by the number of reflectors $L$.

\section{CONClusion And Future Work}

The normalized EC achieved by employing FIRS to support UAV based communications was derived taking into account imperfect phase compensation. The phase compensation error was modeled using von Mises distribution, and the capacity was derived for $L=1$ and 2 in closed-forms, whereas numerical integration and CLT were applied when $L=3$ and $L \geq 4$, respectively. The obtained results demonstrated that capacity degradation due to phase errors is inversely proportional to SNR, which is more apparent for large $L$ values. Interestingly, the capacity deterioration is negligible for $\kappa \geq 6$. It was also shown that increasing $L$ enhances the capacity even when the phase compensation is not ideal. Moreover, it was proven that the effect of the Rician factor $K$ becomes negligible when $K \geq 15$ dB.

The study of UAVs mobility effects on the achievable EC is an interesting future extension to the current work.

\section{REFERENCES}

[1] N. Tafintsev et al., "Aerial access and backhaul in mmWave B5G systems: Performance dynamics and optimization," IEEE Commun. Mag., vol. 58, no. 2, pp. 93-99, Feb. 2020.

[2] C. Yan, L. Fu, J. Zhang, and J. Wang, "A comprehensive survey on UAV communication channel modeling," IEEE Access, vol. 7, pp. 107769$107792,2019$.

[3] Y. Huo, X. Dong, T. Lu, W. Xu, and M. Yuen, "Distributed and multilayer UAV networks for next-generation wireless communication and power transfer: A feasibility study," IEEE IoT J., vol. 6, no. 4, pp. 7103-7115, Aug. 2019.

[4] B. Du, R. Xue, L. Zhao, and V. Leung, "Coalitional graph game for air-to-air and air-to-ground cognitive spectrum Sharing," IEEE Trans. Aerosp. Electron. Syst., vol. 56, no. 4, pp. 2959-2977, Aug. 2020.

[5] M. Al-Jarrah, A. Al-Dweik, E. Alsusa, Y. Iraqi, and M.-S. Alouini, "IRS-assisted UAV communications with imperfect phase compensation," submitted to IEEE Trans. Wireless Commun. [Online]. Available: https://doi.org/10.36227/techrxiv.13153211.v1

[6] E. Basar et al., "Wireless communications through reconfigurable intelligent surfaces," IEEE Access, vol. 7, pp. 116753-116773, 2019.

[7] M. Al-Jarrah, A. Al-Dweik, E. Alsusa, and M.-S. Alouni, "Performance analysis of wireless mesh backhauling using intelligent reflecting surface," IEEE Trans. Wireless Commun.. Early access, doi: 10.1109/TWC.2021.3052370.

[8] W. Shi, X. Zhou, L. Jia, Y. Wu, F. Shu, and J. Wang, "Enhanced secure wireless information and power transfer via intelligent reflecting surface," IEEE Commun. Lett., Early access, doi: 10.1109/LCOMM.2020.3043475.

[9] T. Van Chien, L. Tu, S. Chatzinotas, and B. Ottersten, "Coverage probability and ergodic capacity of intelligent reflecting surface-enhanced communication systems," IEEE Commun. Lett., vol. 25, no. 1, pp. 6973, Jan. 2021.

[10] S. Zhang and R. Zhang, "Capacity characterization for intelligent reflecting surface aided MIMO communication," IEEE J. Sel. Areas Commun., vol. 38, no. 8, pp. 1823-1838, Aug. 2020.

[11] D. Li, "Ergodic capacity of intelligent reflecting surface-assisted communication systems with phase errors," IEEE Commun. Lett., vol. 24, no. 8, pp. 1646-1650, Aug. 2020.

[12] J. Sánchez, P. Ramírez-Espinosa and F. López-Martínez, "Physical layer security of large reflecting surface aided communications with phase errors," IEEE Wireless Commun. Lett., doi: 10.1109/LWC.2020.3029816.

[13] M. Badiu and J. Coon, "Communication through a large reflecting surface with phase errors," IEEE Wireless Commun. Lett., vol. 9, no. 2, pp. 184-188, Feb. 2020.

[14] T. Shafique, H. Tabassum and E. Hossain, "Optimization of wireless relaying with flexible UAV-borne reflecting surfaces," IEEE Trans. Commun., vol. 69, no. 1, pp. 309-325, Jan. 2021.

[15] Y. Maghsoodi and A. Al-Dweik, "Error-rate analysis of FHSS networks using exact envelope characteristic functions of sums of stochastic signals," IEEE Trans. Veh. Technol., vol. 57, no. 2, pp. 974-985, Mar. 2008.

[16] D. W. Matolak and R. Sun, "Unmanned aircraft systems: Air-ground channel characterization for future applications," IEEE Veh. Technol. Mag., vol. 10, no. 2, pp. 79-85, Jun. 2015.

[17] D. W. Matolak and R. Sun, "Air-ground channel characterization for unmanned aircraft systems-Part I: Methods, measurements, and models for over-water settings," IEEE Trans. Veh. Technol., vol. 66, no. 1, pp. 26-44, Jan. 2017.

[18] S. Zhang and R. Zhang, "Intelligent reflecting surface aided multiuser communication: Capacity region and deployment strategy," arXiv Preprint. Online. [Available:] https://arxiv.org/abs/2009.02324

[19] A. Prudnikov, Yu. A. Brychkov, and O. Marichev, Integrals and Series, vol. 1 Elementary Functions. Gordon and Breach Science Publishers, UK, 3rd edition, 1986.

[20] V. Aalo, G. Efthymoglou, and C. Chayawan, "On the envelope and phase distributions for correlated gaussian quadratures," IEEE Commun. Lett., vol. 11 , no. 12 , pp. 985-987, Dec. 2007. 